\title{
Angiotensin-Converting Enzyme Inhibitor Induced Angioedema Occurring after 8 Years of Taking Lisinopril: A Case Report
}

\author{
Wade Jodeh*, Gregory Stone \\ Saint Joseph Mercy Health System, Ann Arbor, MI, USA \\ Email: *wadejodeh@gmail.com
}

How to cite this paper: Jodeh, W. and Stone, G. (2020) Angiotensin-Converting Enzyme Inhibitor Induced Angioedema Occurring after 8 Years of Taking Lisinopril: A Case Report. Case Reports in Clinical Medicine, 9, 115-121.

https://doi.org/10.4236/crcm.2020.95017

Received: April 3, 2020

Accepted: April 19, 2020

Published: April 22, 2020

Copyright $\odot 2020$ by author(s) and Scientific Research Publishing Inc. This work is licensed under the Creative Commons Attribution International License (CC BY 4.0).

http://creativecommons.org/licenses/by/4.0/

\begin{abstract}
Angiotensin-converting enzyme inhibitor induced angioedema (AIIA) can vary from mild to life-threatening. A vast majority of cases of AIIA occur within a month of starting an angiotensin-converting enzyme-inhibitor (ACE-I). We present a 48-year-old male who presented with respiratory failure secondary to AIIA, after being on lisinopril for over 8 years. He had no previous complications secondary to lisinopril and aside from smoking, carried no risk factors for AIIA. Despite conventional treatment for angioedema, he had a prolonged stay in the Medical Intensive Care Unit (MICU). Following discharge, there hasn't been a recurrence of AIIA since the discontinuation of lisinopril. The case is intended to caution that AIIA remains possible even late into a chronic regimen of ACE-I. This is a risk that shouldn't be neglected, even with sparse risk factors or longer duration of ACE-I use. Conventional treatment is not currently in line with proposed etiologies of AIIA. We advocate for more clinical trials involving pharmaceutical agents targeting bradykinin accumulation.
\end{abstract}

\section{Keywords}

Angioedema, Angiotensin Converting Enzyme Inhibitor, Lisinopril, Eight Years

\section{Background}

Angioedema is a localized swelling of subepithelial tissues. It's benign when limited to skin but can cause respiratory distress when present in the mouth, pharynx or larynx [1] [2]. It's estimated that over 40 million people around the world are prescribed ACE-Is (angiotensin-converting enzyme inhibitors), with 
the most common being lisinopril [2] [3]. Drug-induced angioedema has implicated multiple classes of drugs, the most common being Angiotensin-converting enzyme-inhibitor induced angioedema (AIIA) [4]. Though subpopulations can vary, there is an estimated incidence of $0.1 \%-0.6 \%$ of AIIA in patients on ACE-I therapy [2].

AIIA can vary greatly in presentation; ranging from mild to life-threatening angioedema requiring intubation and mechanical ventilation (MV). The highest frequency of AIIA is within the first month of starting ACE-I, particularly the first week, but rare afterward. However, there have been case reports of angioedema occurring 2 years after initiation of therapy [4] [5].

We present a case of AIIA requiring MV and prolonged stay in the Medical Intensive Care Unit (MICU). This case is unique as the patient had been taking lisinopril for over 8 years for hypertension therapy. The case demonstrates that ACE-Is can carry a risk for AIIA for many years. This case also cautions that traditional management of angioedema is not necessarily targeted at the etiology of AIIA.

\section{Case Presentation}

We present a 48-year-old male who was admitted to the MICU with AIIA. He has a long-standing history of hypertension and had been on lisinopril $20 \mathrm{mg}$ for over 8 years. He had been initially started on lisinopril-hydrochlorothiazide 20 $\mathrm{mg}-25 \mathrm{mg}$, then de-escalated to lisinopril nearly 3 years later. His medical history includes Type 2 Diabetes Mellitus, long term insulin use, Chronic Kidney Disease Stage IV, Coronary Artery Disease, Obstructive Sleep Apnea, and Bipolar 1 Disorder. Regarding his social history, he had a 15-pack year smoking history, along with a history of cocaine use for which he had been drug free for 5 years. He lived with his mother and was independent of all activities of daily living. He had not had any recent travels, insect/animal bites or medication changes. Regarding allergies, he had documented severe allergic reaction when exposed to contrast medium over a year prior. His family history was free of any angioedema or anaphylaxis. Prior to presentation the patient had been taking lisinopril daily and had eight pills left over from his last refill.

The patient was at his baseline state of health on the day of presentation. $\mathrm{He}$ had his typical breakfast at 12:30 PM consisting of fried eggs, biscuits, gravy and orange juice. An hour later he started having tongue swelling, which he was able to tolerate for 30 minutes before he started struggling to breathe. His mother realized he was not able to speak and called 911. On presentation in the Emergency Department (ED), there was prominent swelling of his tongue and pharyngeal edema. His oropharynx was completely occluded by his tongue causing respiratory failure. He was promptly intubated and placed on MV, with propofol being used for sedation. A Chest x-ray [see "Figure 1"], confirmed proper placement of endotracheal tube and no evidence of infection contributing to the cause of the patient's respiratory failure (i.e. pneumonia). There was no evidence 
of effusions, lobar consolidation or pneumothorax. He was given epinephrine $0.3 \mathrm{mg}$ intramuscularly, methylprednisolone $125 \mathrm{mg}$ intravenously, along with 3 liters of intravenous fluid and admitted to the MICU. The following day, he was started on diphenhydramine $50 \mathrm{mg}$ three times a day and methylprednisolone was deescalated to $60 \mathrm{mg}$ every 6 hours. On day 3 the patient failed his spontaneous breathing trial (SBT) and respiratory status deteriorated; $\mathrm{FiO}_{2}$ requirement increased from $40 \%$ to $60 \%$, and positive end-expiratory pressure increased from 10 to $12 \mathrm{mmHg}$. The patient's angioedema resolved on Day 4 per physical exam, but the CXR that day [see "Figure 2"] demonstrated worsening

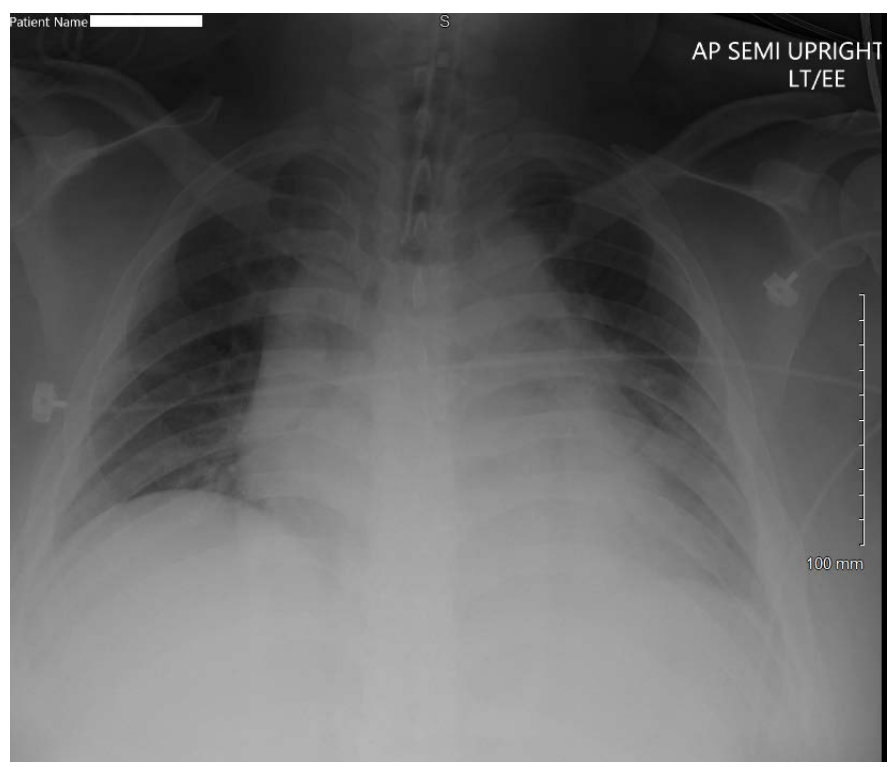

Figure 1. Chest x-ray on admission. Endotracheal tube tip seen $2.9 \mathrm{~cm}$ above the level of the carina. Central bilateral perihilar bronchovascular markins likely related to intubation and atelectasis. No effusions or pneumothorax evident.

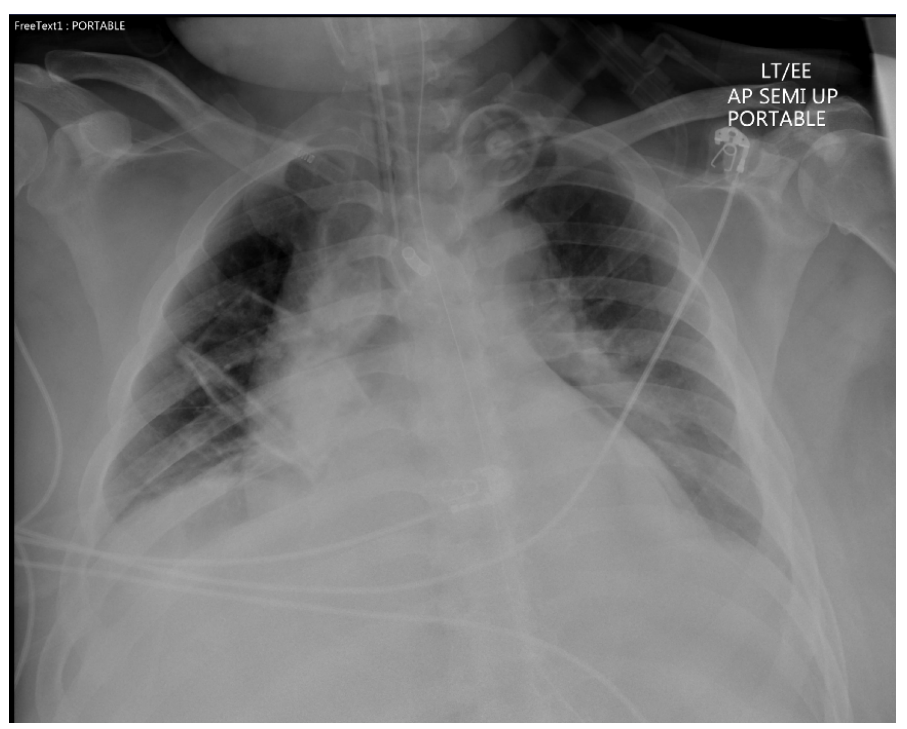

Figure 2. Chest $\mathrm{x}$-ray on hospital day 4. New bibasilar opacities seen, relating to ventilator-associated pneumonia. 
of bilateral opacities and the patient was started on broad-spectrum antibiotics for ventilator-associated pneumonia (VAP). Respiratory cultures later grew Methicillin-resistant staphylococcus aureus (MRSA) and Moraxella catarrhalis and antibiotics were deescalated to vancomycin and ceftriaxone. On day 5 patient was tachypneic and in respiratory distress and was prone-ventilated (requiring multiple staff members to assist) for ARDS (Acute Respiratory Distress Syndrome). On day 9, our patient failed his spontaneous awakening trial but could squeeze my fingers on command.

It wasn't until day 11 that he was extubated, and was able to answer questions with writing. The following day he was able to speak/answer questions and was cleared by speech therapy for solid food. He was subsequently transferred out of the MICU on day 13 in a hemodynamically stable state and speaking comfortably. While in intermediate care, he was recommended Subacute Rehabilitation by both physical and occupational therapy at discharge.

Following discharge, patient has permanently discontinued lisinopril. He has been able to control his hypertension with amlodipine $10 \mathrm{mg}$. Following 18 months of follow up, he has not had any recurrences of angioedema or hospitalizations.

\section{Discussion}

IIA was initially reported as have an incidence of approximately $0.1 \%$ in patients on an ACE-I, however, a prospective trial involving 25,000 patients shows that this value has risen to $0.7 \%$, with incidence higher in certain subpopulations [6]. AIIA has most often occurs within a week to a month of starting ACE-I therapy [5]. Our case stands unique in that our patient has been compliant with his lisinopril for over 8 years, since the initial diagnosis of hypertension. With our literature review, the only reported duration of ACE-I usage prior to AIAI longer than that of our patient was 11 years [4]. Otherwise, there have been multiple reports of AIIA after 2 years of ACE-I use. This emphasizes that the temporal relationship between ACE-I and AIIA is not always close. AIIA carries a higher risk in African Americans, age older than 65, those regularly on aspirin, those on Non-steroidal anti-inflammatory drugs (NSAIDs), smokers and those with previous drug/seasonal allergies [2] [7] [8] [see "Table 1"]. Our patient, was Caucasian and in his 40s with no prior ACE-I induced cough, allergies to aspirin or NSAIDs, $\mathrm{C} 1$ esterase deficiency (or family history of. The only risk factor he had for AIIA was smoking. This further raised our awareness that AIIA should not be neglected, despite scare risk factors and duration.

Different etiologies and mechanisms for AIIA have been described, with many centered around ACE and bradykinin physiology. ACE exists within the lining of our lungs, endothelial and renal epithelial cells [9].

ACE metabolizes bradykinin, which is a potent vasodilator. Bradykinin is converted from Kallikrein by $\mathrm{C} 1$ esterase. With ACE-I use, both bradykinin and Substance $\mathrm{P}$ do accumulate, as their inactivation is inhibited. This accumulation 
Table 1. Sociodemographic characteristics and risk factors regarding AIIA.

\begin{tabular}{ccc}
\hline Characteristics and Risk Factors & Our Patient & Average AIIA Patient \\
\hline Age & 48 years & $>65$ years \\
Onset of AIIA from Starting ACE-I Therapy & $>8$ years & Within 1 - 4 weeks \\
Gender & Male & Female \\
History of Prior Angioedema & Absent & Present \\
Seasonal Allergies & Absent & Present \\
Aspirin/NSAID Use & Absent & Present \\
Family History of Angioedema & Absent & Present \\
Tobacco Use History & Present & Present \\
\hline
\end{tabular}

results in vasodilation/vascular permeability, which is postulate to manifest in AIIA [10] [11] [12]. In patients with angioedema, the absence of itching or urticaria should draw attention to diagnosis of AIIA. The presence of urticaria likely implicates a different group of etiologies such as infectious, IgE-mediated allergic cases and direct mast cell activation [13].

The increase in the frequency of AIIA has made it all the clearer that there is a dearth of standardized treatment. Current standard treatment includes glucocorticoids, antihistamines, and epinephrine. All of these agents work against AIIA's inflammatory response well, they do not work on the bradykinin system [9]. This is problematic because if elevated bradykinin/Substance $\mathrm{P}$ activity is responsible for the mechanism of AIIA, standardized management would not be helpful against underlying etiology [9] [14].

Our patient was managed with traditional therapy, and his angioedema did not resolve until day 4 of hospitalization. In a multicenter review of 108 patients with angioedema, only 5 patients took $>48$ hours to respond to conventional treatment [15]. Interestingly, our patient's angioedema did not resolve sooner. We speculate our patient would have benefited from bradykinin targeted therapy, as he may have had an earlier resolution of angioedema and avoided ARDS complicating and prolonging his stay.

The Food and Drug Administration has approved icatibant for acute AIIH and hereditary angioedema. This drug functions on the bradykinin mechanism by competitively antagonizing bradykinin B2 receptors [16]. Data regarding its use has been mixed. Positive outcomes and resolution of symptoms within 10 hours administration of subcutaneous icatibant were shown in 12/13 case reports/series [17]. However, a more recent randomized controlled trial of 121 subjects failed to demonstrate the efficacy of icatibant compared to placebo [18]. Further research is warranted regarding the efficacy of icatibant and other agents inhibiting bradykinin mediated angioedema. Optimistically speaking, icatibant could have resolved our patient's angioedema, preventing ARDS (and subsequent prolonged MICU stay). In addition to icatibant, ecallantide, tranexamic acid, purified $\mathrm{C} 1$ inhibitor concentrate, and possibly fresh frozen plasma are other 
therapies that are effective for aborting attacks of angioedema in hereditary angioedema (HAE). In theory, these treatments could be helpful in ACE inhibitor-induced angioedema, but available studies are greatly limited [19].

\section{Conclusions}

- This is a case report of ACE-I induced angioedema occurring after the patient had been taking lisinopril for over 8 years. Providers should be aware that patients on ACE-Is continue to carry a risk of angioedema years into chronic treatment. This is a risk that shouldn't be neglected, even with sparse risk factors or longer duration of ACE-I use.

- ACE-Is should be discontinued immediately following AIIA without re-challenging the patient. An alternative for blood pressure control should be discussed with patients

- There is no definitive etiology of AIIA. Though most theories explaining AIIA are postulated around the accumulation of bradykinin.

- No consistently efficacious treatment for AIIA exists. More trials need to be conducted with agents targeting the bradykinin system for treating AIIA.

\section{Conflicts of Interest}

The authors declare no conflicts of interest regarding the publication of this paper.

\section{References}

[1] Taber, C.W. and Thomas, C.L. (2005) Tabers Cyclopedia Medical Dictionary. F.A. Davis Co., Philadelphia.

[2] Byrd, J.B., Adam, A. and Brown, N.J. (2006) Angiotensin-Converting Enzyme Inhibitor-Associated Angioedema. Immunology and Allergy Clinics of North America, 26, 725-737. https://doi.org/10.1016/j.iac.2006.08.001

[3] Kostis, W.J., Shetty, M., Chowdhury, Y.S. and Kostis, J.B. (2018) ACE Inhibitor-Induced Angioedema: A Review. Current Hypertension Reports, 20, 55. https://doi.org/10.1007/s11906-018-0859-x

[4] Norman, J.L., Holmes, W.L., Bell, W.A. and Finks, S.W. (2012) Life-Threatening ACE Inhibitor-Induced Angioedema after Eleven Years on Lisinopril. Journal of Pharmacy Practice, 26, 382-388. https://doi.org/10.1177/0897190012465990

[5] Vleeming, W., Amsterdam, J.G.V., Stricker, B.H. and Wildt, D.J.D. (1998) ACE Inhibitor-Induced Angioedema. Incidence, Prevention and Management. Drug Safety, 18, 171-188. https://doi.org/10.2165/00002018-199818030-00003

[6] Hedner, T., Samuelsson, O., Lunde, H., Lindholm, L., Andren, L. and Wiholm, B.E. (1992) Angio-Oedema in Relation to Treatment with Angiotensin Converting Enzyme Inhibitors. BMJ, 304, 941-946. https://doi.org/10.1136/bmj.304.6832.941

[7] Kostis, J.B., Kim, H.J., Rusnak, J., Casale, T., Kaplan, A., Corren, J., et al. (2005) Incidence and Characteristics of Angioedema Associated with Enalapril. Archives of Internal Medicine, 165, 1637. https://doi.org/10.1001/archinte.165.14.1637

[8] Kamil, R.J., Jerschow, E., Loftus, P.A., Tan, M., Fried, M.P., Smith, R.V., et al. (2016) Case-Control Study Evaluating Competing Risk Factors for Angioedema in a 
High-Risk Population. The Laryngoscope, 126, 1823-1830. https://doi.org/10.1002/lary.25821

[9] Lieberman, P., Nicklas, R.A., Oppenheimer, J., Kemp, S.F., Lang, D.M., Bernstein, D.I., et al. (2010) The Diagnosis and Management of Anaphylaxis Practice Parameter: 2010 Update. Journal of Allergy and Clinical Immunology, 126, 477-480.e1-42. https://doi.org/10.1016/j.jaci.2010.06.022

[10] Adam, A., Cugno, M., Molinaro, G., Perez, M., Lepage, Y. and Agostoni, A. (2002) Aminopeptidase $\mathrm{P}$ in Individuals with a History of Angio-Oedema on ACE Inhibitors. The Lancet, 359, 2088-2089. https://doi.org/10.1016/S0140-6736(02)08914-6

[11] Campo, P., Fernandez, T.D., Canto, G. and Mayorga, C. (2013) Angioedema Induced by Angiotensin-Converting Enzyme Inhibitors. Current Opinion in Allergy and Clinical Immunology, 13, 337-344.

https://doi.org/10.1097/ACI.0b013e328362b835

[12] Hoover, T., Lippmann, M., Grouzmann, E., Marceau, F. and Herscu, P. (2009) Angiotensin Converting Enzyme Inhibitor Induced Angio-Oedema: A Review of the Pathophysiology and Risk Factors. Clinical \& Experimental Allergy, 40, 50-61.

https://doi.org/10.1111/j.1365-2222.2009.03323.x

[13] Asero, R. (2020) New-Onset Urticaria [Internet]. https://www.uptodate.com/contents/new-onset-urticaria?search=etiologiesofurticar ia\&source $=$ machineLearning\&graphicRef $=54872$ graphicRef 54872

[14] Beltrami, L., Zingale, L.C., Carugo, S. and Cicardi, M. (2006) Angiotensin-Converting Enzyme Inhibitor-Related Angioedema: How to Deal with It. Expert Opinion on Drug Safety, 5, 643-649. https://doi.org/10.1517/14740338.5.5.643

[15] Chiu, A.G., Burningham, A.R., Newkirk, K.A., Krowiak, E.J., Davidson, B.J. and Deeb, Z.E. (2001) Angiotensin-Converting Enzyme Inhibitor-Induced Angioedema: A Multicenter Review and an Algorithm for Airway Management. Annals of Otology, Rhinology \& Laryngology, 110, 834-840. https://doi.org/10.1177/000348940111000906

[16] Zuraw, B.L., Bernstein, J.A., Lang, D.M., Craig, T., Dreyfus, D., Hsieh, F., et al. (2013) A Focused Parameter Update: Hereditary Angioedema, Acquired C1 Inhibitor Deficiency, and Angiotensin-Converting Enzyme Inhibitor-Associated Angioedema. Journal of Allergy and Clinical Immunology, 131, 1491-1493. https://doi.org/10.1016/j.jaci.2013.03.034

[17] Illing, E.J., Kelly, S., Hobson, J.C. and Charters, S. (2012) Icatibant and ACE Inhibitor Angioedema. Case Reports, 2012, pii: bcr2012006646. https://doi.org/10.1136/bcr-2012-006646

[18] Sinert, R., Levy, P., Bernstein, J.A., Body, R., Sivilotti, M.L., Moellman, J., et al. (2017) Randomized Trial of Icatibant for Angiotensin-Converting Enzyme Inhibitor-Induced Upper Airway Angioedema. The Journal of Allergy and Clinical Immunology: In Practice, 5, 1402-1409.e3. https://doi.org/10.1016/j.jaip.2017.03.003

[19] Hirschy, R., Shah, T., Davis, T. and Rech, M.A. (2018) Treatment of Life-Threatening ACE-Inhibitor-Induced Angioedema. Advanced Emergency Nursing Journal, 40, 267-277. https://doi.org/10.1097/TME.0000000000000211 